\title{
Studies of surface two-dimensional photonic band-gap structures
}

\author{
A. W. Cross, ${ }^{\text {a) }}$ I. V. Konoplev, A. D. R. Phelps, and K. Ronald \\ Department of Physics, University of Strathclyde, Glasgow, G4 ONG, United Kingdom
}

(Received 1 July 2002; accepted 30 October 2002)

Two-dimensional (2D) surface photonic band-gap (SPBG) structures can be obtained by providing a shallow corrugation of the inner surface of a waveguide wall. It can be used as a distributed mirror, a cavity, or a filter in integrated optics or microwave electronics. These structures can also be an alternative to conventional 2D PBG or 1D Bragg structures. In this article, we present the results of theoretical and experimental studies of 2D SPBG structures. Data obtained from experiments are compared with theoretical results and good agreement between theory and experiment is demonstrated. Comparison of a coaxial 2D SPBG structure with a conventional 1D Bragg structure is also presented. (C) 2003 American Institute of Physics. [DOI: 10.1063/1.1531816]

\section{INTRODUCTION}

Extensive study of the phenomenon of wave propagation in periodic structures has led to many technological breakthroughs in conventional optics and vacuum electronics. In 1968, Kovalev et al. ${ }^{1}$ suggested the use of an onedimensional shallow corrugation (1D Bragg corrugation) on the wall of a waveguide to obtain mode transformation in the microwave frequency range and in 1977, Yariv and Nakamura considered the use of a 1D Bragg corrugation for integrated optics applications. ${ }^{2}$ Bratman et al. ${ }^{3}$ suggested that $1 \mathrm{D}$ Bragg structures could be used to obtain distributed feedback in the cavity of free-electron masers (FEM) and cyclotron autoresonance masers (CARM). In 1987, it was predicted that a $3 \mathrm{D}$ periodicity of refractive index may result in the appearance of a forbidden band gap for electromagnetic radiation ${ }^{4}$ and shortly after this prediction it was experimentally proven. ${ }^{5}$ In recent years, many successful experiments were carried out on FEMs, CARMs, and conventional lasers with distributed feedback, using 1D periodic structures. ${ }^{6-13}$ However, for high-power FEM and CARM microwave oscillator experiments, where single-mode operation was realized, the output power achieved was less than $50 \mathrm{MW}$. The power limitation was due to the restriction in the transverse dimensions of the cavity (for example, its diameter), which did not exceed the operating wavelength by more than a few wavelengths. ${ }^{14}$ Further increase of the output power without increasing the transverse size of the cavity may result in $\mathrm{rf}$ breakdown inside the interaction space and consequently interruption of the microwave generation. ${ }^{15}$ On the other hand, the increase of the transverse dimension of the 1D Bragg cavity leads to the loss of its selectivity and consequently to the loss of single-mode operation. ${ }^{14}$ To overcome these problems the use of two-dimensional (2D) distributed feedback, which can be realized due to wave scattering on a $2 \mathrm{D}$ double-periodic corrugation has been suggested. ${ }^{16}$ A number of theoretical works dedicated to the use of such feedback in high-power microwave devices were published in Refs. 1620. Preliminary experiments with an FEM using planar struc-

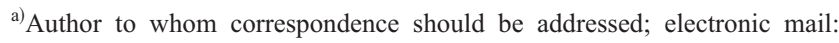
cabs72@strath.ac.uk tures with $2 \mathrm{D}$ corrugation have been completed. ${ }^{21}$ In contrast with the conventional 2D photonic band-gap (PBG) structures which are usually machined from either long dielectric or metals rods in air or air ${ }^{22-25}$ tubes in dielectric materials, the $2 \mathrm{D}$ structures can be obtained either by providing shallow 2D corrugations on the waveguide walls, or using a dielectric material with a refractive index which has a double periodicity. ${ }^{26}$ Thus, in order to distinguish these structures from conventional 2D PBG structures we will refer to it as "2D surface photonic band-gap (SPBG) structures." It is important to note that in the works ${ }^{16-20}$ the structures discussed were referred to simply as 2D Bragg structures by analogy with 1D Bragg structures. We also decided to use the new term to underline that the $2 \mathrm{D}$ SPBG structure is a $2 \mathrm{D}$ PBG structure and not a modification of a 1D Bragg structure.

In contrast to a $1 \mathrm{D}$ Bragg structure where two counterpropagating waves are directly coupled, ${ }^{1-3}$ in an ideal $2 \mathrm{D}$ SPBG structure there are four waves participating in the scattering. The forward and backward waves are indirectly coupled via a second pair of transverse waves. ${ }^{16-20}$ The presence of the additional energy fluxes, transverse with respect to the direction of propagation of the wave interacting with the medium, act to synchronize the radiation from the different parts of the large active medium. It is important to note that the existence of the $2 \mathrm{D}$ scattering on the $2 \mathrm{D}$ corrugation results in the appearance of a forbidden band gap, which is specific for each type of wave of the waveguide. Let us also note that the structures are compatible with any active medium including a high-current relativistic electron beam. These robust SPBG structures have high-rf breakdown strength and their location on the surface of the waveguide should ensure easy cooling which is important for any highpower laser application. They are also much easier to fabricate in comparison with conventional 2D PBG structures, and in the case of integrated optics ${ }^{2}$ should allow a significant decrease of the cavity size in comparison with conventional 2D PBG structures and 1D Bragg mirrors. The possible applications for these structures are narrow-band microwave and optical filters, all-optical transistors, selective elements for high-power laser cavities, and optical and microwave multiplexers. 


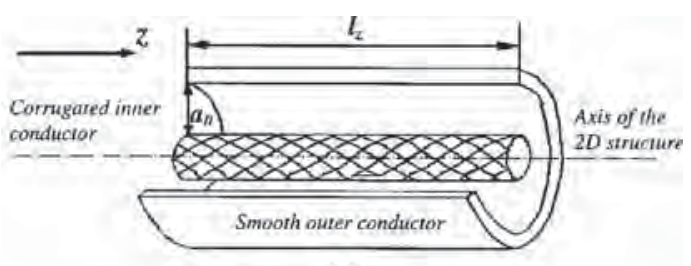

(a)

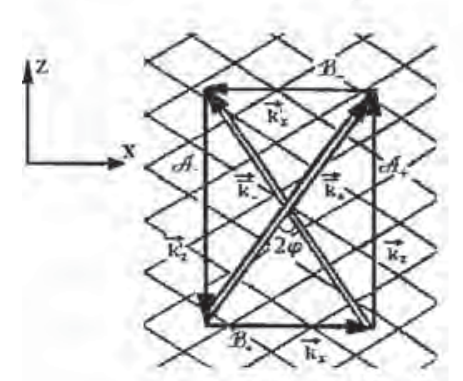

(b)

FIG. 1. (a) Schematic diagram of a 2D SPBG coaxial structure with a corrugated inner conductor. (d) Schematic diagram of a two-dimensional distributed feedback loop realized on an ideal 2D corrugation.

This article is devoted to the theoretical and experimental study of coaxial 2D SPBG structures with different patterns. To describe the field evolution we used coupled wave theory, which was very successfully applied to describe the field evolution in 1D periodic structures. ${ }^{1-3}$ In Sec. II, a coaxial 2D SPBG with an ideal sinusoidal corrugation is considered. The reflection and transmission coefficients from such a structure were found and analyzed. In Sec. III, 2D SPBG structures with different patterns are considered and compared with an ideal corrugation. In Sec. IV, the experimental setup used for the cold microwave measurements of the 2D SPBG structures is presented and the experimental results obtained are compared with the theoretical predictions. For the conclusion, we summarize the results obtained and discuss possible problems, which can be subjects for future research.

\section{IDEAL COAXIAL 2D SPBG STRUCTURE}

Let us consider a coaxial 2D SPBG structure consisting of a smooth outer and a corrugated inner conductor of radii $r_{\text {out }}, r_{\text {in }}$, respectively, and of length $l_{z}$ [Fig. 1(a)]. The corrugation of the outside surface of the inner conductor can be represented by the following expression:

$$
r=r_{\text {in }}+a_{1}\left[\cos \left(\overline{k_{z}} z\right) \cos (\bar{m} \varphi)\right],
$$

where $a_{1}$ is the corrugation depth, $\overline{k_{z}}=2 \pi / d_{z}, d_{z}$ is the period of corrugation along the $z$ coordinate, and $\bar{m}$ is the number of the corrugation variations along the azimuthal coordinate $\varphi$. It is assumed that the corrugation of the inner conductor is shallow, i.e., $\overline{k_{x, z}} a_{1} \ll 1$ and $a_{1} \ll \lambda$ where $\overline{k_{x}}$ $=\bar{m} / r_{0}$, and $\lambda$ is the radiation wavelength. It was mentioned in the Introduction that a medium with the double periodic refractive index (see, for example, Ref. 26) $n=n_{0}$ $+\left[n_{1} \cos \left(\overline{k_{z}} z\right) \cos (\bar{m} \varphi)\right]$ can also be used instead of the shallow corrugation where $n_{1} \ll n_{0}$. In Fig. 1(a), the lines on the surface of the conductor indicate the crests of the corrugations and the crossings of the lines correspond to the centers of the two-dimensional grating. Assuming that the radii $r_{\text {in }}$ and $r_{\text {out }}$ greatly exceed the radiation wavelength $\lambda$ and the distance between the conductors $a_{0}=r_{\text {out }}-r_{\text {in }}$, i.e.,

$$
r_{\text {in,out }} \gg a_{0}, r_{\text {in,out }} \gg \lambda,
$$

the dispersion equation for the eigenwaves of the coaxial waveguide can be reduced to the following form: ${ }^{27}$

$$
k^{2}=\frac{\omega^{2}}{c^{2}} \cong k_{z}^{2}+k_{\varphi}^{2}+k_{r}^{2},
$$

where $\omega$ is the wave frequency, $c$ is the speed of light, $k_{z}$ is the longitudinal wave number, $k_{\varphi}=M / r_{0}$ is the azimuthal wave number, $r_{0}=\left(r_{\text {in }}+r_{\text {out }}\right) / 2, k_{r}=p \pi / a_{0}$ is the radial wave number, and $M$ and $p$ are the azimuthal and radial variation indices. The dispersion equation is similar to that obtained for the eigenwaves of the planar waveguide. This allows one to neglect the small curvature of the cavity surface and adopt the planar coordinate system. Let us introduce also the new transverse coordinate $x=r_{0} \times \varphi$ instead of the azimuthal coordinate $\varphi$ and use $k_{x}$ instead of $k_{\varphi}$, i.e., $k_{x}$ $\equiv k_{\varphi}$.

The field inside the 2D SPBG structure can be presented in the form of four coupled waves: $\mathcal{A}_{ \pm}$propagating in the $\pm z$ and $\mathcal{B}_{ \pm}$propagating in the $\pm x$ directions (compare with Refs. 1-3 and see also Refs. 16-20,26);

$$
\begin{aligned}
\vec{E}= & \operatorname{Re}\left\{\left[\vec{E}_{b}^{0}(r)\left(\mathcal{B}_{+}(x, z) e^{-i k_{x} x}+\mathcal{B}_{-}(x, z) e^{i k_{x}^{\prime} x}\right)+\vec{E}_{a}^{0}(r)\right.\right. \\
& \left.\left.\times\left(\mathcal{A}_{+}(x, z) e^{-i k_{z} z}+\mathcal{A}_{-}(x, z) e^{i k_{z}^{\prime} z}\right)\right] e^{i \omega t}\right\}
\end{aligned}
$$

Here, $\mathcal{A}_{ \pm}(x, z), \mathcal{B}_{ \pm}(x, z)$ are the slow functions of the $x$ and $z$ coordinates, $k_{x}, k_{x}^{\prime}$ and $k_{z}, k_{z}^{\prime}$ are the transverse and longitudinal wave numbers of the partial waves $\mathcal{B}_{+}, \mathcal{B}_{-}$and $\mathcal{A}_{+}, \mathcal{A}_{-}$respectively, $E_{a, b}^{0}(r)$ are functions describing the spatial wave profile along the $r$ coordinate, which coincides with one of the eigenmodes of the coaxial waveguide. Due to the circular geometry of the coaxial system the wave amplitudes should satisfy the following cyclic boundary conditions:

$$
\mathcal{B}_{ \pm}\left(x+l_{x}, z\right)=\mathcal{B}_{ \pm}(x, z), \mathcal{A}_{ \pm}\left(x+l_{x}, z\right)=\mathcal{A}_{ \pm}(x, z),
$$

where $l_{x}=2 \pi r_{0}$ is the cavity mean circumference. These conditions allow the partial wave amplitudes $\mathcal{A}_{ \pm}(x, z)$, $\mathcal{B}_{ \pm}(x, z)$ to be represented in the following Fourier series:

$$
\begin{aligned}
& \mathcal{A}_{ \pm}(x, z)=\sum_{m=-\infty}^{\infty} \mathcal{A}_{ \pm}^{m}(z) e^{i m s x}, \\
& \mathcal{B}_{ \pm}(x, z)=\sum_{m=-\infty}^{\infty} \mathcal{B}_{ \pm}^{m}(z) e^{i m s x},
\end{aligned}
$$

where $s=2 \pi \mathcal{A}_{x}$, and to consider each Fourier term as an eigenmode of the waveguide with an azimuthal variation index equal to either $m$ for the partial waves $\mathcal{A}_{ \pm}$or $M_{ \pm}^{\prime}=m$ $\mp|M|$ in case of the partial waves $\mathcal{B}_{ \pm}$, respectively.

Let us assume for simplicity that $\overline{k_{x}}=\overline{k_{z}}=\bar{k}$ and $\vec{k}_{ \pm}$ $=\vec{k}\left(\vec{x}_{0} \pm \vec{z}_{0}\right)$, where $\vec{x}_{0}$ and $\vec{z}_{0}$ are the unit vectors along the 
$x$ and $z$ coordinates and $\overline{k_{x}}, \overline{k_{z}}$ are the amplitudes of the projections of the lattice eigenvectors $\vec{k}_{ \pm}$on the axes $x$ and $z$. Formation of a two-dimensional feedback loop via 2D scattering of the partial waves on the corrugation is presented in Fig. 1(b). ${ }^{17,20}$ In this figure, the partial wave $\mathcal{A}_{+}$propagating in the $+z$ direction is scattered into waves $\mathcal{B}_{ \pm}$propagating in the transverse $\pm x$ directions and scattering into waves $\mathcal{A}_{ \pm}$ which ensures that the two-dimensional feedback loop $\mathcal{A}_{+}$ $\rightarrow \mathcal{B}_{ \pm} \rightarrow \mathcal{A}_{-} \rightarrow \mathcal{B}_{ \pm} \rightarrow \mathcal{A}_{+}$is completed. To obtain an efficient coupling of the partial waves $\mathcal{A}_{ \pm} \leftrightarrow \mathcal{B}_{ \pm}$, the following Bragg resonance conditions ${ }^{1-3}$ should be satisfied for each pair of coupled waves:

$$
\begin{aligned}
& \vec{k}_{z}-\vec{k}_{x}=\vec{k}_{-} ; \vec{k}_{z}^{\prime}-\vec{k}_{x}^{\prime}=-\vec{k}_{-} \\
& \vec{k}_{z}-\vec{k}_{x}^{\prime}=\vec{k}_{+} ; \vec{k}_{z}^{\prime}-\vec{k}_{x}=-\vec{k}_{+}
\end{aligned}
$$

However, the 2D-feedback loop can only be obtained when conditions (7) are fulfilled simultaneously. Therefore the four partial waves undergo the coupling on the $2 \mathrm{D}$ structure if the following resonance conditions are satisfied (see, also, the Appendix):

$$
k_{z}=k_{z}^{\prime} \cong \overline{k_{z}}, k_{x}=k_{x}^{\prime} \cong \overline{k_{x}}(|\bar{m}|=|M|) .
$$

For the corrugation considered in Eq. (1) $\bar{m}$ can be either positive or negative. As a result, the azimuthal variation indices of the partial waves propagating in the $\pm x$ directions are equal to $M_{+}^{\prime}=[|\bar{m}|+|m| \operatorname{sgn}(m)]$ and $M_{-}^{\prime}=-[|\bar{m}|$ $-|m| \operatorname{sgn}(m)]$, respectively. Let us assume that $\mathcal{A}_{ \pm}^{m}$ $=\mathcal{A}_{ \pm}^{-m}, \mathcal{B}_{ \pm}^{m}=\mathcal{B}_{ \pm}^{-m}$ and further in the text the sign of the index $m$ will solely indicate either an up shift $(+)$ or a down shift $(-)$ of the wave frequency from the exact Bragg frequency. Taking into account Eq. (8), we note that for the corrugation considered the radial variation index of the partial waves will be conserved, i.e., for example the TEM mode of the coaxial waveguide can only be scattered into a $\mathrm{TE}_{m, 0}^{-}$mode. It is important to note that the mutual scattering of the partial wave $\mathcal{A}_{+}$into the waves $\mathcal{B}_{ \pm}$should ensure the synchronization of radiation from different parts of the active medium.

The field scattering on the corrugation, when conditions (8) are satisfied, can be described by the following set of coupled wave equations (compare with Ref. 26) for the dimensionless amplitudes $A_{ \pm}, B_{ \pm}$(for more details, see the Appendix):

$$
\begin{aligned}
& \pm \frac{\partial A_{ \pm}}{\partial z}+i \delta A_{ \pm}+\sigma A_{ \pm}+i \alpha\left(B_{+}+B_{-}\right)=0, \\
& \pm \frac{\partial B_{ \pm}}{\partial x}+i \delta B_{ \pm}+\sigma B_{ \pm}+i \alpha\left(A_{-}+A_{+}\right)=0,
\end{aligned}
$$

where $A_{ \pm}=\mathcal{A}_{ \pm} e^{\mp i \delta z}, B_{ \pm}=\mathcal{B}_{ \pm} e^{\mp i \delta x}, \delta=\left(\omega-\omega_{0}\right) / c$ is the frequency detuning from the Bragg resonance, $\omega_{0}$ $=c \sqrt{\overline{k^{2}}+k_{r}^{2}}$ is the Bragg frequency, $\sigma$ is the distributed ohmic losses, $\alpha$ is the wave-coupling coefficient, and $k_{r}$ is the radial wave number. We also suppose that $\left|\omega-\omega_{0}\right|$ $\ll \omega_{0}$. In accordance with Refs. 1 and 3 the wave-coupling coefficient depends on the field structure of the coupled modes. The expression for $\alpha$ in the set of Eqs. (9), if only one conductor is corrugated, can be represented in the following form. ${ }^{17,18}$

$$
\alpha=\frac{a_{1} k}{4 a_{0}} f,
$$

where $f$ is the parameter which is determined by the coupled waves' field structures. For example, if the following coupling $\mathrm{TE}_{m, 0} \leftrightarrow \mathrm{TE}_{M^{\prime}, 0}$ takes place $f=\left(1-\left|m / M^{\prime}\right|\right)$ and under the condition $|m| \ll\left|M^{\prime}\right|$ we obtain $f \cong 1$. It is also important to note that $f=1$ for the $\mathrm{TEM} \leftrightarrow \mathrm{TE}_{m, 0}^{-} \quad$ scattering. Following ${ }^{1-3,17}$ the parameter $f$, can be found for other pairs of coupled partial waves. Let us note that if instead of the corrugation a dielectric material with a $2 \mathrm{D}$ biperiodic refractive index is laid on the waveguide wall the coupling coefficient can be obtained with a simple substitution of $a_{1}$ to $n_{1}$ and $a_{0}$ to $n_{0}$ (compare with Ref. 26).

Substituting Eq. (6) in the set of Eqs. (9), the following relation between the partial waves can be obtained:

$$
B_{+}^{m}+B_{-}^{m}=\frac{-2 i \alpha(i \delta+\sigma)\left(A_{+}^{m}+A_{-}^{m}\right)}{(i \delta+\sigma)^{2}+s^{2} m^{2}}
$$

and the set of Eqs. (9) can be reduced to

$$
\pm \frac{d A_{ \pm}^{m}}{d z}+\frac{2 \alpha^{2}(i \delta+\sigma)}{(i \delta+\sigma)^{2}+s^{2} m^{2}}\left(A_{+}^{m}+A_{-}^{m}\right)+(i \delta+\sigma) A_{ \pm}^{m}=0 .
$$

The reflection and transmission coefficients from such a structure can be found by taking into account the following boundary conditions:

$$
A_{+}^{m}(z=0)=A_{0}^{m}, A_{-}^{m}\left(z=l_{z}\right)=0,
$$

where $l_{z}$ is the length of the 2D structure. Considering conditions (13) the analytic expressions for the reflection $R_{m}$ and the transmission $T_{m}$ coefficients can be obtained from Eq. (12) as functions of the azimuthal index $m$ and frequency detuning $\delta_{0}$ of the incident wave. Let us note that $\delta_{0}$ is real, in contrast to $\delta$ which is complex.

$$
\begin{aligned}
& R_{m}=\frac{\overline{\lambda_{m}^{2}}-p_{m}^{2}}{q_{m}\left[p_{m}-\overline{\lambda_{m}} c t g\left(\overline{\lambda_{m}} l_{z}\right)\right]}, \\
& T_{m}=\frac{-i \overline{\lambda_{m}}}{\sin \left(\overline{\lambda_{m}} l_{z}\right)\left[p_{m}-i \overline{\lambda_{m}} c \operatorname{tg}\left(\overline{\lambda_{m}} l_{z}\right)\right]},
\end{aligned}
$$

where

$$
q_{m}=\frac{2 \alpha^{2} \widetilde{\delta}}{s^{2} m^{2}+\widetilde{\delta}^{2}}, \quad p_{m}=\widetilde{\delta}\left(\frac{2 \alpha^{2}}{s^{2} m^{2}+\widetilde{\delta}^{2}}+1\right), \tilde{\delta}=i \delta_{0}+\sigma,
$$

and

$$
\overline{\lambda_{m}}=-i \widetilde{\delta}\left(\frac{4 \alpha^{2}}{\widetilde{\delta}^{2}+s^{2} m^{2}}+1\right)^{1 / 2}
$$

where $\sigma$ is associated with the rf power losses. The reflection coefficient can be transformed to the following form: 


$$
R_{m}=\frac{-2 \alpha}{2 \alpha^{2}+\left(s^{2} m^{2}-\widetilde{\delta}^{2}\right)-i\left(\left(s^{2} m^{2}-\widetilde{\delta}^{2}\right)\left[4 \alpha^{2}+\left(s^{2} m^{2}-\widetilde{\delta}^{2}\right)\right]\right)^{1 / 2} \operatorname{ctg}\left[\widetilde{\delta} l_{z}\left(\frac{4 \alpha^{2}+\left(s^{2} m^{2}-\widetilde{\delta}^{2}\right)}{s^{2} m^{2}-\widetilde{\delta}^{2}}\right)^{1 / 2}\right]}
$$

Analyzing expression (17) we have to note that for each mode with index $m$ such a structure provides an effective reflection zone (forbidden band gap), Fig. 2, inside a frequency interval defined by the condition $\operatorname{Re}\left(\overline{\lambda_{m}^{2}}\right) \leqslant 0 .{ }^{20}$ Considering zero rf power losses, i.e., $\sigma=0$ the boundaries of each forbidden band gap (reflection zone) can be found and presented in the following form:

$$
s m \leqslant\left|\delta_{0}\right| \leqslant\left(4 \alpha^{2}+s^{2} m^{2}\right)^{1 / 2} \text {. }
$$

As was shown in Ref. 20 and as is evident from Fig. 2, the width of the gaps decreases with an increase of $m$. The maxima of the reflection coefficients are located at exact Bragg frequencies [defined by Eq. (8)] and strongly depend on the parameter $\alpha / \sigma .{ }^{20}$ In contrast to a $1 \mathrm{D}$ Bragg mirror where $R \sim \tanh \left(\alpha l_{z}\right),{ }^{2,3}$ the length parameter does not affect the maximum amplitude of the reflection coefficients. By adjusting the parameter $\alpha l_{z}$, however, the effective width of the reflection zones can be controlled while maintaining a high- (up to $100 \%$ ) reflection coefficient at the precise Bragg frequency. These features of the 2D SPBG structure allow one to control independently the maximum amplitude and width of the reflection zone and should allow one to obtain a narrow frequency band mirror, or filter. It is important to

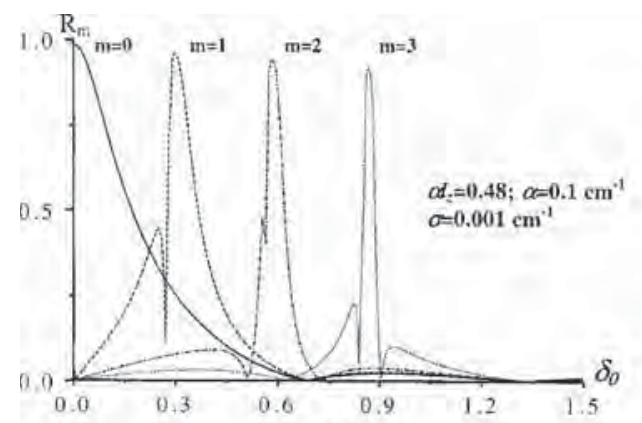

(a)

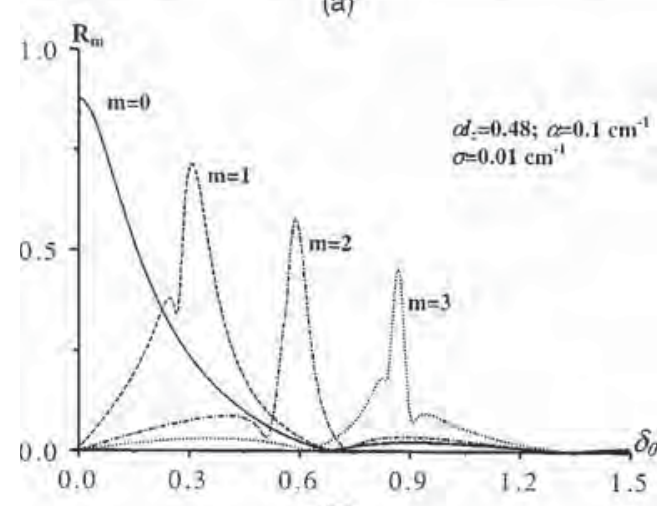

(b)

FIG. 2. The reflection coefficient $R_{m}$ vs frequency detuning $\delta_{0}$ when: $s$ $=0.29 \mathrm{~cm}^{-1}, \alpha=0.1 \mathrm{~cm}^{-1}, \alpha l_{z}=0.48 \mathrm{~m} \in[0 ; 2]$, and (a) $\sigma=0.001 \mathrm{~cm}^{-1}$ and (b) $\sigma=0.01 \mathrm{~cm}^{-1}$. note that in a real system, the rf power losses are always present, while the coupling coefficient, which is proportional to the corrugation depth, can be easily adjusted. Note that the incident wave on the 2D structure with an azimuthal index $m$ is reflected into a wave with the same azimuthal index $m$ and the frequency locations of the maxima of the reflection coefficients for the modes with different spatial structures are different. Assuming also $\sigma \neq 0, \alpha \neq 0$, and $\alpha / \sigma>1$ it is important to note that the drop of the reflection coefficient with a decrease of $\alpha / \sigma$ is more dramatic for incident waves with $m \neq 0$, as compared with an azimuthally symmetric mode, Fig. 2(b). Therefore, if the structure is used in a two-mirror cavity as one of the mirrors, then by optimizing the parameter $\alpha / \sigma$ the selectivity of the cavity over the wave azimuthal index $m$ can be maintained, even if the transverse dimension of the cavity grossly exceeds the operating wavelength. ${ }^{20}$ In Fig. 3, the profiles of the partial waves at the exact Bragg frequency are presented for three different values of $\alpha / \sigma$, when the azimuthal-symmetric wave is incident on the 2D structure. Thus, when $\alpha / \sigma \gg 1$ the absolute values of the amplitudes of the waves $\mathcal{A}_{+}$and $\mathcal{A}_{-}$are nearly equal and $|R| \rightarrow 1$. A decrease of the parameter $\alpha / \sigma \sim 1$ [Fig. $3(\mathrm{~b})]$ leads to a significant drop of the amplitude of the reflected $\mathcal{A}_{-}$wave and an increase of the amplitude of the transmitted wave $\mathcal{A}_{+}\left(z=l_{z}\right) \sim 0.5$. When $\alpha / \sigma \ll 1$ [Fig. 3(c)] the amount of energy which passes from one partial wave into another due to scattering $(\alpha \neq 0)$ is insufficient as compared to the energy dissipated and the maximum reflection coefficient at the exact Bragg resonance frequency is of the order $\sim O(\alpha / \sigma)^{2}$. As a result, the condition $\alpha \approx \sigma$ can be considered as the lower limit in the minimum value of the coupling coefficient, i.e., this dictates the minimum amplitude of corrugation, or minimum amplitude of variation of the refractive index. This also limits the minimum effective width of the reflection zone as defined by the coupling coef-

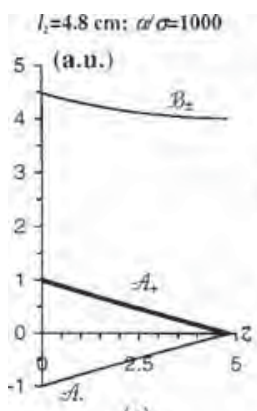

(a)

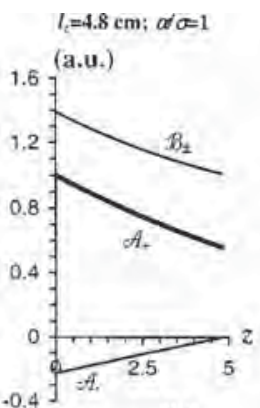

(b)

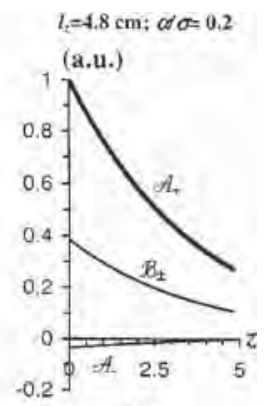

(c)
FIG. 3. Spatial profiles of the partial waves $\mathcal{A}_{ \pm}$and $\mathcal{B}_{ \pm}$. The partial wave $\mathcal{A}_{+}$is the incident wave of unit amplitude (solid wide line) on the 2D SPBG structure: $l_{z}=4.8 \mathrm{~cm}, s=0.29 \mathrm{~cm}^{-1}$, and $\alpha / \sigma$ is (a) 1000 ; (b) 1 ; and (c) 0.2 . 

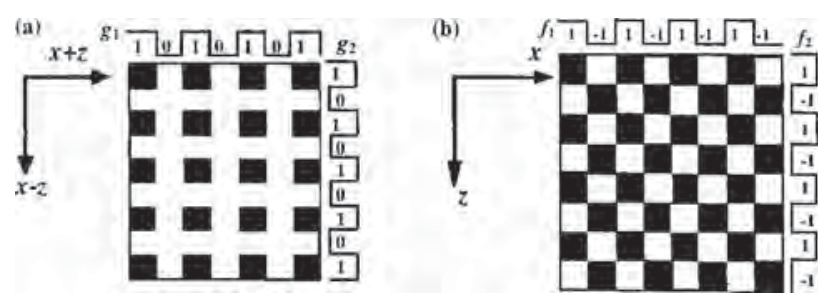

FIG. 4. Schematic diagram of the 2D corrugations with (a) "rhombus" and (b) "chessboard" pattern.

ficient (18). It is important to note that a similar but stronger condition also exists for the full 3D PBG structures and will be one of the subjects for future work.

\section{2D SPBG STRUCTURE WITH DIFFERENT CORRUGATION PATTERNS}

The machining of the "ideal" corrugation as presented in Fig. 1 is difficult and therefore it is very attractive to find a substitute for an "ideal" sinusoidal corrugation. A 2D "square wave" corrugation can be a viable alternative to the sinusoidal corrugation. In the following figures [Figs. 4(a) and 4(b)] the schematic diagrams of the surface with "rhombus" [Fig. 4(a)] and "chessboard" [Fig. 4(b)] patterns are presented. Both 2D corrugated surfaces can be described as the product of two periodic functions: ${ }^{20}$

$$
a=\left(\begin{array}{l}
f_{1}\left(\overline{k_{\xi}} \xi\right) f_{2}\left(\overline{k_{\eta}} \eta\right) \\
g_{1}\left(\overline{k_{\xi}} \xi\right) g_{2}\left(\overline{k_{\eta}} \eta\right)
\end{array}\right.
$$

where

$$
\begin{aligned}
& g_{i}(\mathrm{~s})=\left(\begin{array}{cc}
0 & 0<\mathrm{s}<d_{\mathrm{s}} / 2 \\
1 & d_{\mathrm{s}} / 2<\mathrm{s}<d_{\mathrm{s}}
\end{array}\right. \text { [Fig. 4(a)], } \\
& f_{i}(\mathrm{~s})=\left(\begin{array}{cc}
-1 & 0<\mathrm{s}<d_{\mathrm{s}} / 2 \\
1 & d_{\mathrm{s}} / 2<\mathrm{s}<d_{\mathrm{s}}
\end{array}[\text { Fig. } 4(\mathrm{~b})],\right.
\end{aligned}
$$

$\mathrm{s}$ is the variable, $i=1,2$ and $d_{\mathrm{s}}$ is the period of the periodic functions $f_{i}\left(\mathrm{~s}+d_{\mathrm{s}}\right)=f_{i}(\mathrm{~s}), g_{i}\left(\mathrm{~s}+d_{\mathrm{s}}\right)=g_{i}(\mathrm{~s})$. The "rhombus" corrugation [Fig. 4(a)] can be obtained by machining $\bar{m}$ fold helical rectangular grooves (in clockwise and counterclockwise directions) on the surface of the conductor. The corrugation with a "chessboard" pattern [Fig. 4(b)] can be obtained by assembling separately machined gears of a width of half a period, or by using micromachining techniques.

Using Fourier expansion the functions $g_{i}$ and $f_{i}$ can be rewritten as

$$
\begin{aligned}
& g_{i}(\mathrm{~s})=1+\frac{4}{\pi} \sum_{n} \frac{(-1)^{n}}{2 n+1} \cos (2 n+1) \frac{2 \pi}{d_{\mathrm{s}}} \mathrm{s}, \\
& f_{i}(\mathrm{~s})=\frac{4}{\pi} \sum_{n} \frac{1}{2 n+1} \sin (2 n+1) \frac{2 \pi}{d_{\mathrm{s}}} \mathrm{s},
\end{aligned}
$$

where $n=0,1,2, \ldots$, and $i=1,2$ is the function number. It is important to note that $\xi=x+z, \eta=x-z$ for the rhombus

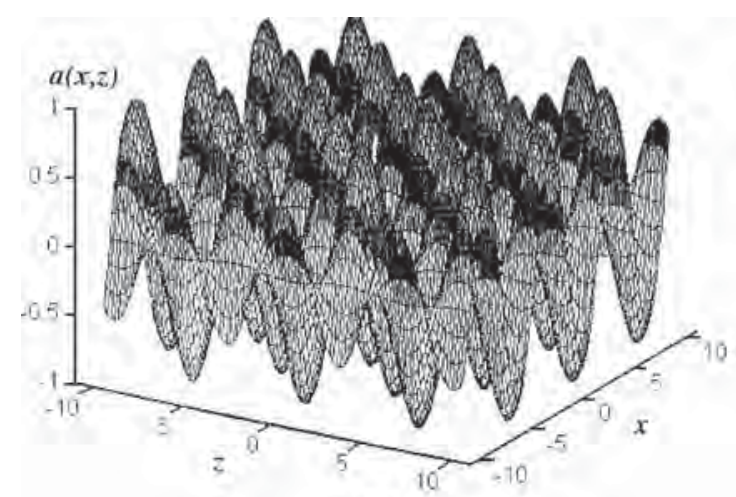

(a)

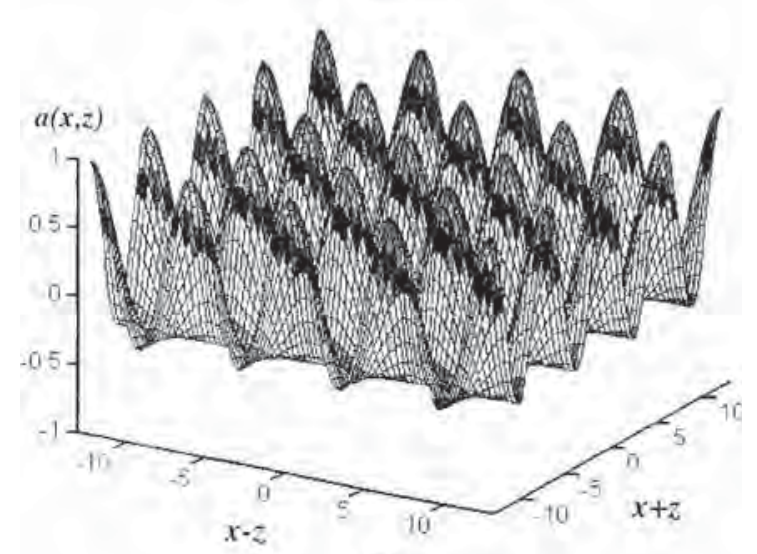

(b)

FIG. 5. 3D representation of $2 \mathrm{D}$ corrugations described by expressions (a) for chessboard pattern (22) and (b) rhombus pattern (23).

pattern and $\xi=x, \quad \eta=z$ for the "chessboard" pattern $(\bar{x}$ $\left.=r_{0} \vec{\varphi}\right)$. Taking into account that the terms of the Fourier expansion are proportional to $\sim[1 /(2 n+1)]$, the higher harmonics, apart from the fundamental, can be neglected and expressions (19) can be approximated as follows:

$$
a(x, z) \cong \frac{8 a_{1}}{\pi^{2}}\left[\cos \left(\overline{k_{x}} x-\overline{k_{z}} z\right)+\cos \left(\overline{k_{x}} x+\overline{k_{z}} z\right)\right]+\cdots
$$

for the surface with a chessboard pattern and

$$
\begin{aligned}
a(x, z) \cong & \frac{a_{1}}{\pi}\left[\cos \left(\overline{k_{x}} x-\overline{k_{z}} z\right)+\cos \left(\overline{k_{x}} x+\overline{k_{z}} z\right)\right] \\
& +\frac{2 a_{1}}{\pi^{2}}\left[\cos \left(2 \overline{k_{x}} x\right)+\cos \left(2 \overline{k_{z}} z\right)\right]+\cdots
\end{aligned}
$$

for the surface with a rhombus pattern, ${ }^{28}$ where $\overline{k_{x, z}}$ $=2 \pi / d_{x, z}$ and $d_{x, z}$ are the corrugation periods along the $x$ and $z$ coordinates and $d_{x}=\bar{m} / r_{0}$ (see, also Ref. 21). The 3D representation of the pattern, which corresponds to the corrugations, described by Eqs. (22) and (23) is shown in Figs. 5(a) and 5(b), respectively, and the zero level corresponds to the unperturbed surface of the waveguide. It is easy to see from Eq. (22) that the "chessboard" corrugation approxi- 

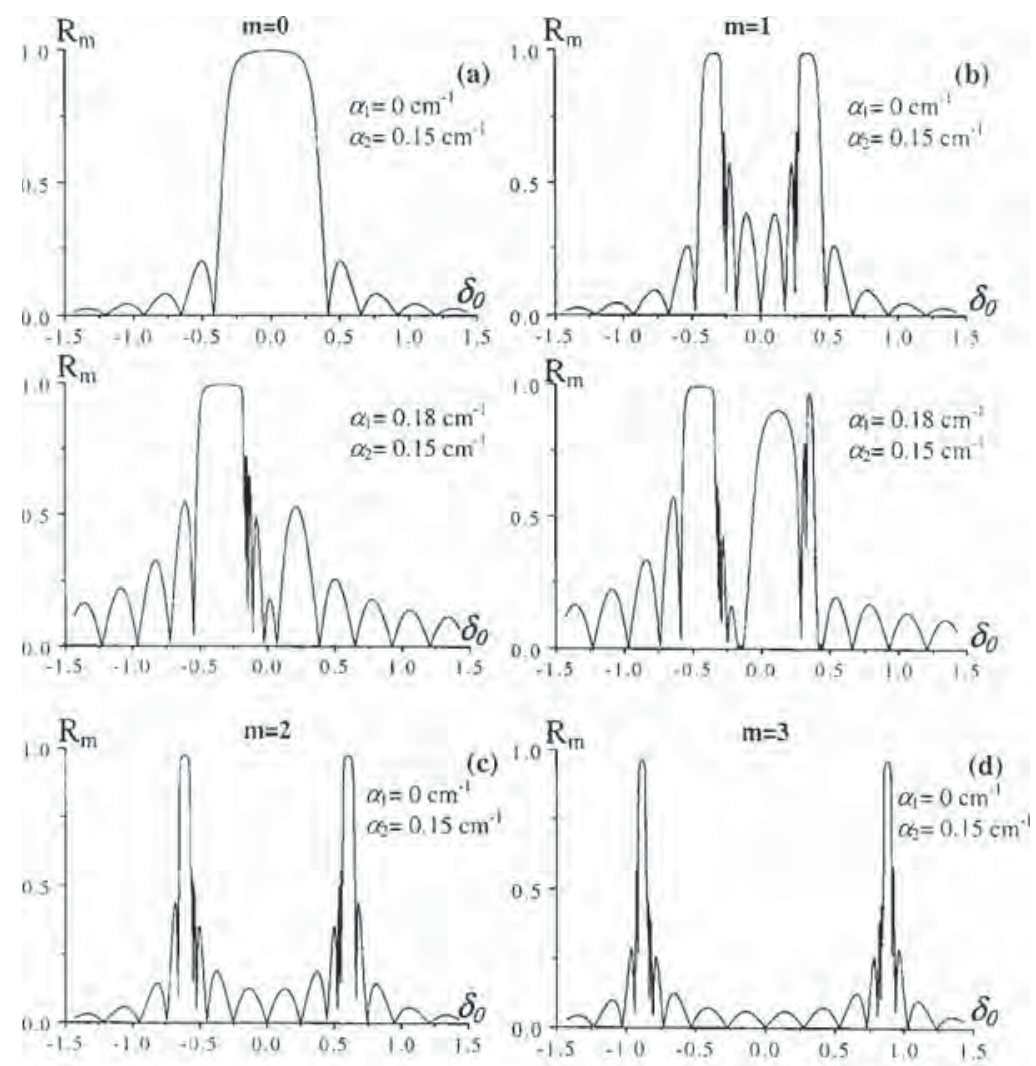

FIG 6. The reflection coefficient from the ideal 2D SPBG structure (top figures) and 2D SPBG structure with a "rhombus" corrugation pattern (bottom figures) when $l_{z}=10 \mathrm{~cm}, s=0.29 \mathrm{~cm}^{-1}, \sigma=0.001 \mathrm{~cm}^{-1}$, and (a) $m=0$, (b) $m=1$, (c) $m=2$, and (d) $m=3$.
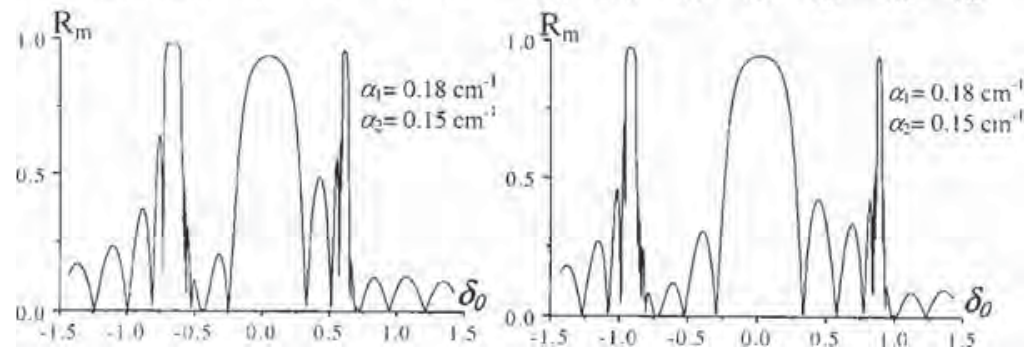

mates well to an "ideal" sinusoidal 2D corrugation. Therefore, it is expected that the properties of the 2D SPBG structure with such a pattern should be similar to those of a $2 \mathrm{D}$ SPBG structure with an "ideal" corrugation.

The "rhombus" corrugation described by Eq. (23) contains 1D (second term of expression proportional to $2 a_{1} / \pi^{2}$ ) and 2D (first term of the expression proportional to $a_{1} / \pi$ ) corrugations. It is interesting to note that a similar $2 \mathrm{D}$ pattern is frequently used to obtain a conventional 2D PBG structure. $^{25}$ The corrugation described by Eq. (23) provides distributed 2D coupling for four partial waves (4) propagating along the $z$ and $x$ coordinates, if the geometrical parameters of the corrugation $\bar{k}_{x, z}$ satisfy the resonance conditions (8), with the longitudinal wavenumbers $k_{z}, k_{z}^{\prime}$ of the partial waves $\mathcal{A}_{+}, \mathcal{A}_{-}$and the azimuthal wave-numbers $k_{x}, k_{x}^{\prime}$ of the partial waves $\mathcal{B}_{+}, \mathcal{B}_{-}$. Simultaneously, the $1 \mathrm{D}$ coupling of the partial waves takes place if the following resonance conditions are satisfied:

$$
2 \overline{k_{z}} \approx k_{z}+k_{z}^{\prime} ; 2 \overline{k_{x}} \approx k_{x}+k_{x}^{\prime},
$$

for coupling-waves $\mathcal{A}_{+} \leftrightarrow \mathcal{A}_{-}$and $\mathcal{B}_{+} \leftrightarrow \mathcal{B}_{-}$, respectively. Obviously conditions (24) are satisfied if conditions (8) are met. However, if Eq. (24) is satisfied condition (8) may not be met. Taking into account the presence of a " $1 \mathrm{D}$ component" in the corrugation the coupled-wave equations can be presented in the following form: ${ }^{28}$

$$
\begin{aligned}
& \pm \frac{\partial A_{ \pm}}{\partial z}+i \delta A_{ \pm}+i \alpha_{1} A_{\mp}+i \alpha_{2}\left(B_{+}+B_{-}\right)=0, \\
& \pm \frac{\partial B_{ \pm}}{\partial x}+i \delta B_{ \pm}+i \alpha_{1} B_{\mp}+i \alpha_{2}\left(A_{-}+A_{+}\right)=0,
\end{aligned}
$$

where $\alpha_{2}$ and $\alpha_{1}$ are the wave-coupling coefficients associated with $2 \mathrm{D}$ and $1 \mathrm{D}$ Bragg scattering, respectively. The coupling coefficient $\alpha_{1}$ is defined in Refs. $1-3$ and 29 while $\alpha_{2}$ is given by formula (10). It is interesting to note that Eqs. (25) describe either 1D Bragg scattering when $\alpha_{2}=0$, or 2D Bragg scattering when $\alpha_{1}=0$. The reflection and transmission coefficient can be found using the same procedure described in Sec. II. The expressions for these coefficients coincide with those presented in Eqs. (14) and (15) if the following substitutions of parameters (16) are conducted: 


$$
\begin{aligned}
& p_{m}= \frac{2 \alpha_{2}^{2} \delta_{+}}{s^{2} m^{2}-\delta_{+} \delta_{-}}+\frac{\delta_{+}+\delta_{-}}{2} ; \\
& q_{m}=\frac{2 \alpha_{2}^{2} \delta_{+}}{s^{2} m^{2}-\delta_{+} \delta_{-}}-\frac{\delta_{+}-\delta_{-}}{2}, \\
& \overline{\lambda_{m}}=\left(\frac{4 \alpha_{2}^{2} \delta_{+}^{2}}{s^{2} m^{2}-\delta_{+} \delta_{-}}+\delta_{+} \delta_{-}\right)^{1 / 2} ; \delta_{ \pm}=\delta-i \sigma \pm \alpha_{1} .
\end{aligned}
$$

The boundaries of the forbidden band gap are also defined by the condition $\operatorname{Re}\left(\overline{\lambda_{m}^{2}}\right) \leqslant 0$ and can be written in the following form:

$$
\begin{aligned}
& \operatorname{Re}\left[4 \alpha_{1}^{2} \delta_{+}^{2}+\delta_{+} \delta_{-}\left(s^{2} m^{2}-\delta_{+} \delta_{-}\right)\right]=0, \\
& \operatorname{Re}\left(s^{2} m^{2}-\delta_{+} \delta_{-}\right)=0 .
\end{aligned}
$$

Considering zero rf power losses and $m=0$, the boundaries of the band gaps can be found. In contrast with the "ideal" sinusoidal 2D structure there are now two asymmetric band gaps down shifted and up shifted from the precise Bragg frequency. The boundaries of these zones are defined by the following expressions:

$$
-\left(2 \alpha_{2}+\alpha_{1}\right) \leqslant \delta \leqslant-\alpha_{1}
$$

for the down shifted and

$$
\begin{aligned}
& \left(2 \alpha_{2}-\alpha_{1}\right) \leqslant \delta \leqslant \alpha_{1}, \text { if } \alpha_{2}<\alpha_{1} \\
& \alpha_{1} \leqslant \delta \leqslant\left(2 \alpha_{2}-\alpha_{2}\right), \text { if } \alpha_{2}>\alpha_{1}
\end{aligned}
$$

for the up-shifted gap. Figure 6 illustrates the results of numerical calculations of the reflection coefficient for $m$ $\in[0 ; 3]$ from the 2D SPBG structure with ideal (top picture) and rhombus (bottom picture) corrugations. When $m$ is small, $m=0,1$, the mutual influence of $1 \mathrm{D}$ and 2D scattering is significant. However, for relatively large $m$, i.e., $m \geqslant 2$, this mutual influence becomes small and the reflection from such a structure can be considered as a linear superposition of the reflection coefficients due to $1 \mathrm{D}$ and $2 \mathrm{D}$ scattering. The location and width of the reflection zones associated with 1D and $2 \mathrm{D}$ scattering are practically unaffected by each other. This makes it difficult to provide mode selection over the azimuthal variation index in an oversized cavity using such a structure as a selective mirror.

\section{EXPERIMENTAL STUDY OF 2D SPBG STRUCTURES}

The experimental setup presented in Fig. 7(a) allows one to measure both the reflection and transmission coefficients in the operating frequency range. The measurements of the transverse electromagnetic energy fluxes in the 2D SPBG structures were also conducted by using a pickup waveguide probe which was attached to the surface of the outer conductor.

For excitation of the structures a TEM-mode of a coaxial waveguide was formed at the input of the Bragg structure. To produce such a wave beam an additional transmission line was constructed, see Fig. 7(b). The input of the transmission line was a single-mode, $\mathrm{Ka}$ band rectangular waveguide $(7.2 \mathrm{~mm} \times 3.8 \mathrm{~mm})$. The transmission line itself was made

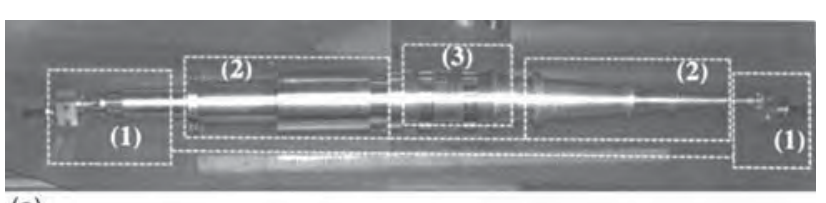

(a)

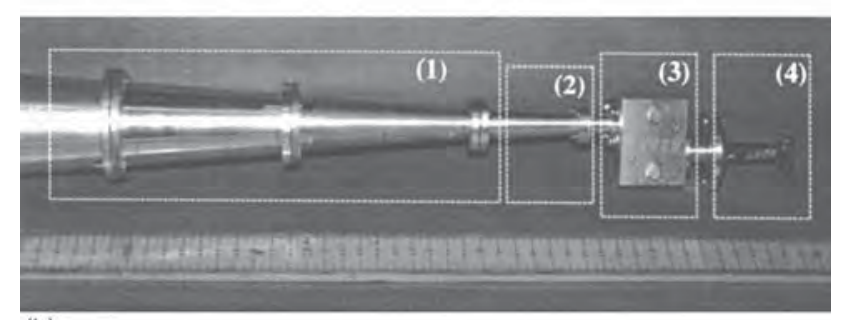

(b)

FIG. 7. (a) Photograph of part of the microwave experimental setup which consists of waveguide mode converters [boxes (1)], adiabatically opening coaxial horns [boxes (2)], and section where the SPBG structure was located [box (3)]. (b) Part of the transmission line which consists of an adiabatically opening horn [box (1)], $\mathrm{TM}_{0,1} \leftrightarrow \mathrm{TEM}$ mode converter [box (2)], $\mathrm{TE}_{1,1} \leftrightarrow \mathrm{TM}_{0,1}$ mode converter [box (3)]; and $\mathrm{TE}_{1,1}$ (circular waveguide) $\leftrightarrow \mathrm{TE}_{1,0}$ (rectangular waveguide) mode converter [box (4)].

from three mode converters, which provide the required mode transformation in the operating frequency band (30-40 $\mathrm{GHz}$ ): from the launched $\mathrm{TE}_{0,1}$ wave of a single-mode rectangular waveguide to the $\mathrm{TE}_{1,1}$ wave of a circular waveguide (the first mode converter) then to a $\mathrm{TM}_{0,1}$ wave of a circular waveguide (the second mode converter), ${ }^{30}$ and finally to a TEM wave of a coaxial waveguide (the third mode converter). ${ }^{31}$ An additional coaxial slowly up-tapered waveguide horn (opening angle of $3^{\circ}$ ) of length $\sim 60 \mathrm{~cm}$ was used to connect from the converters to the oversized coaxial structure. The output dimensions of the coaxial waveguide at the connection with the Bragg structure were as follows: diameters of $5.9 \mathrm{~cm}$ of the inner conductor and $7.9 \mathrm{~cm}$ of the outer conductor.

The 2D SPBG structures with the "chessboard" pattern were made of lengths $l_{z}=4.8$ and $10 \mathrm{~cm}$, Fig. 8, with the corrugation depth of $0.08 \mathrm{~cm}$, which corresponds to $\alpha$ $\approx 0.12 \mathrm{~cm}^{-1}$. The corrugation was made on the outer surface of the inner conductor of the coaxial waveguide with inner and outer radii $r_{\text {in }}=2.95 \mathrm{~cm}$ and $r_{\text {out }}=3.90 \mathrm{~cm}$, respectively. The corrugations were made with the number of azimuthal

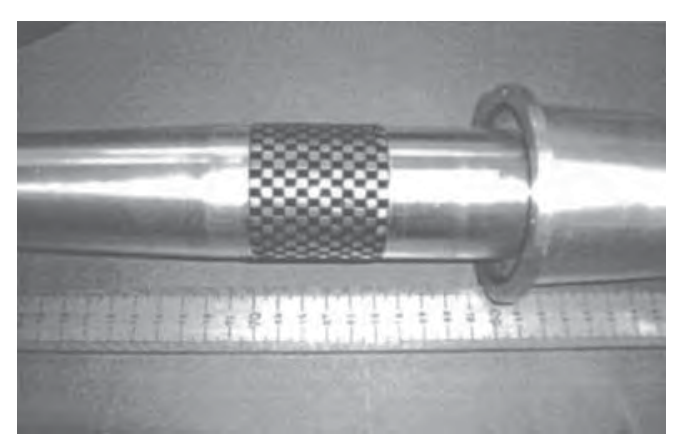

FIG. 8. Photograph of the 2D corrugated inner conductor of the 2D SPBG coaxial structure with chessboard corrugation pattern. 


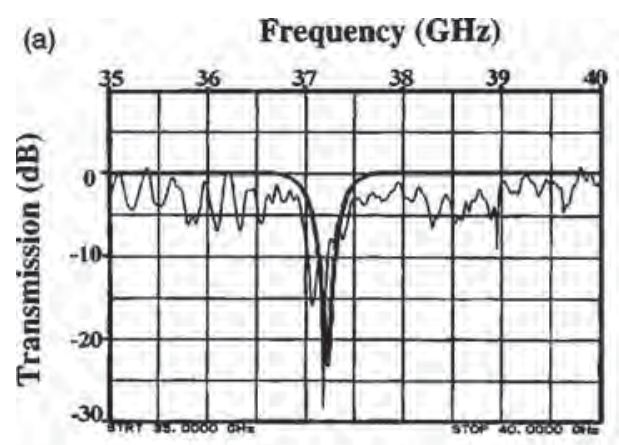

(b)

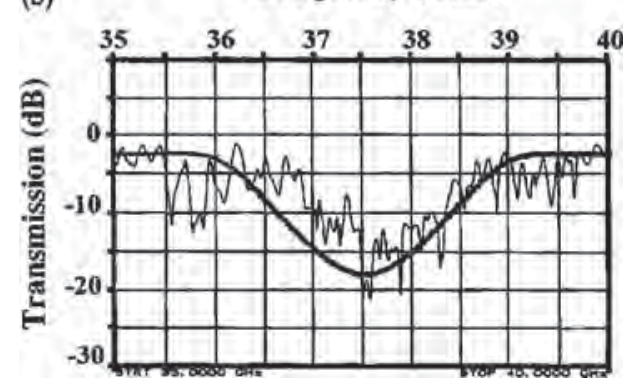

FIG. 9. The transmission coefficient from a $2 \mathrm{D}$ SPBG structure with the "chessboard" pattern when (a) $l_{z}=4.8 \mathrm{~cm}$ and (b) $10 \mathrm{~cm}, \alpha \cong 0.12 \mathrm{~cm}^{-1}$, $\sigma \approx 0.001 \mathrm{~cm}^{-1}$, and the Bragg frequencies are $\sim 37.3$ and $\sim 37.5 \mathrm{GHz}$, respectively.

variations $\bar{m}=24$. The first structure of $10 \mathrm{~cm}$ length had a period $d_{z}=0.800 \mathrm{~cm}$ and the second structure of $4.8 \mathrm{~cm}$ length had $d_{z}=0.804 \mathrm{~cm}$. The periods correspond to the frequencies of the precise resonances (Bragg frequencies) of 37.5 and $37.3 \mathrm{GHz}$, for the 10 and $4.8 \mathrm{~cm}$ long structures, respectively. The parameters of the corrugation were chosen to provide coupling between TEM and $\mathrm{TE}_{24,0}$ waves. In Fig. 9(a) and (b) the transmission coefficients from the 2D SPBG structures of length $l_{z}=4.8$ and $10 \mathrm{~cm}$, respectively, versus frequency of the incident wave are presented in the frequency band from 35.0 to $40.0 \mathrm{GHz}$. From Fig. 9, it is clear that the minimum of the transmission coefficients does not depend on the length of the SPBG structure and is around $\sim-20 \mathrm{~dB}$. This confirms the theoretical results obtained for the "ideal" sinusoidal 2D SPBG structure as shown in Fig. 9.

To compare the $1 \mathrm{D}$ and the $2 \mathrm{D}$ structures, the $1 \mathrm{D}$ Bragg structures of length 30 and $5 \mathrm{~cm}$ with the same corrugation period of $0.4 \mathrm{~cm}$ and corrugation depth $a_{1}=0.015 \mathrm{~cm}$ were studied. In Fig. 10, the measured transmission coefficients are presented. Together with the experimental result the theoretical prediction is also plotted and is shown by the thick solid line. In contrast with the results obtained for the 2D SPBG structure with "chessboard" corrugation, two distinctive reflection zones corresponding to $\mathrm{TEM} \leftrightarrow \operatorname{TEM}(\alpha$ $\left.\approx 0.08 \mathrm{~cm}^{-1}\right)$ and TEM $\leftrightarrow \mathrm{TM}_{0.1}\left(\alpha \approx 0.11 \mathrm{~cm}^{-1}\right)$ wave scattering were observed and the dependence of the maximum of the amplitude of the transmission coefficients on the coupling coefficient and the length of the structure $\left[|T|^{2} \approx 1\right.$ $\left.-\tanh ^{2}\left(\alpha l_{z}\right)\right]$ is also clearly evident. Another specific feature of the $2 \mathrm{D}$ scattering in comparison with the 1D scattering is that coupling between forward and backward waves (for in-

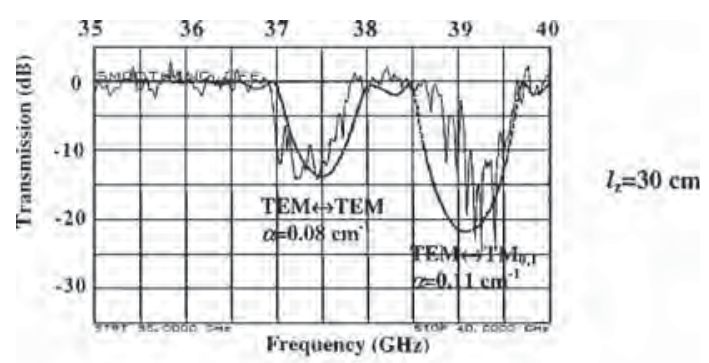

(a)

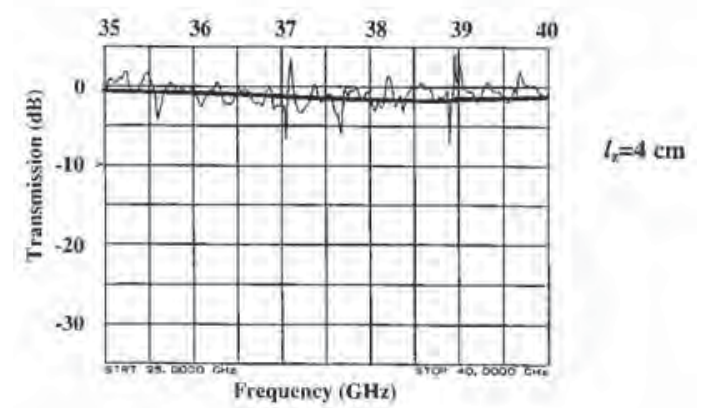

(b)

FIG. 10. Theoretical (thick-solid line) and experimental (thin-solid line) frequency dependencies of the transmission coefficient from a coaxial 1D Bragg structure when $\bar{k}=15.7 \mathrm{~cm}^{-1}, r_{\text {in }}=3.02 \mathrm{~cm}, r_{\text {out }}=3.95 \mathrm{~cm}$, and (a) $l_{z}=30 \mathrm{~cm}$ and (b) $l_{z}=5 \mathrm{~cm}$.

stance, $\mathcal{A}_{ \pm}$) takes place solely via their mutual scattering into waves propagating in the azimuthal direction $\mathcal{B}_{ \pm}$. To conduct measurements of the transverse electromagnetic energy fluxes (associated with partial waves $\mathcal{B}_{ \pm}$) a slit in the outer conductor wall was made to allow a standard singlemode rectangular waveguide probe to be attached to the cavity. It was possible to locate the waveguide at different angles to the slit and locate the probe either radially or tangentially with respect to the surface of the outer conductor. The transverse electromagnetic fluxes were only measured, as opposed to a background noise signal, when the rectangular waveguide probe was positioned tangentially to the waveguide. In Fig. 11, the result of the measurement of the transverse fluxes (thin line) and the theoretical prediction (bold line) are presented for the 2D SPBG structure of a length of $4.8 \mathrm{~cm}$. Such fluxes were not measured when the 2D SPBG

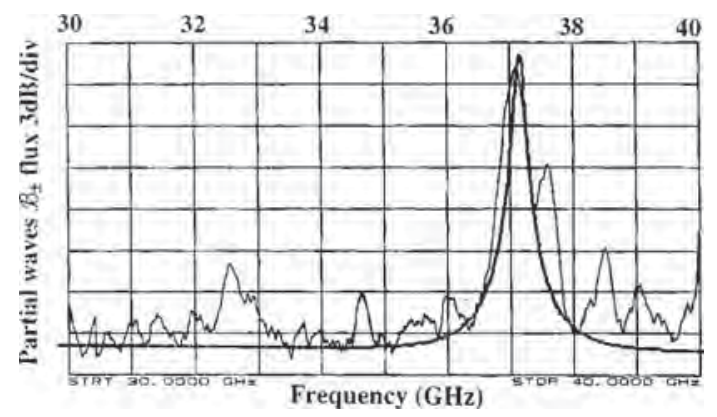

FIG. 11. Theoretical (thick-solid line) and experimental (thin-solid line) frequency dependencies of scattering of partial waves $\mathcal{A}_{ \pm}$into partial waves $\mathcal{B}_{ \pm}$when $l_{z}=4.8 \mathrm{~cm}, \alpha \cong 0.12 \mathrm{~cm}^{-1}, \sigma \approx 0.001 \mathrm{~cm}^{-1}$, and the Bragg frequency $\sim 37.3 \mathrm{GHz}$. 
structure was substituted with either a smooth conductor, or a 1D Bragg structure. The existence of transverse $\mathrm{rf}$ power fluxes is important if the structure is to be used to synchronize radiation from different parts of an oversized active medium, or to obtain a narrow-band filter.

\section{CONCLUSION}

In this article, the "ideal" coaxial 2D surface photonic band-gap structure has been theoretically studied. The coupled-wave theory used to describe such a structure has been presented and coupled wave equations have been obtained. The reflection and transmission coefficients from such a structure were found and the influence of the ohmic losses on these coefficients have been analyzed and discussed. Considering the 2D SPBG structure with a "rhombus" pattern it is shown that such a structure should provide both $1 \mathrm{D}$ and 2D Bragg scattering and as a result it cannot be used to provide mode selection over the wave azimuthal index. The use of a $2 \mathrm{D}$ biperiodic dielectric ${ }^{26}$ instead of a biperiodic corrugation has been considered. Using the results obtained above it is easy to foresee that if a nonlinear medium (such as a Kerr medium) with a double periodic refractive index is used when $n_{1}$ is dependent on the field intensity, the nonlinear change of the width of the band gap due to a change of the coupling coefficient (ratio $n_{1} / n_{0}$ ) while still maintaining high reflection at the exact resonance frequency, as well as induced transparency, may be observed. The theory also predicts that a band gap will appear only when the coupling coefficient, i.e., the ratio $n_{1} / n_{0}$ is larger than some threshold value, which is defined by distributed losses inside the structure. The set of equations describing the field evolution inside the structure should be presented in a timedependent form with a modified coupling coefficient, i.e.,

$$
\begin{aligned}
& \left(\frac{\partial}{\partial Z}+\frac{\partial}{\partial \tau}\right) A_{+}+\sigma A_{+}+i \hat{\alpha}(I)\left(B_{+}+B_{-}\right)=0, \\
& \left(-\frac{\partial}{\partial Z}+\frac{\partial}{\partial \tau}\right) A_{-}-\sigma A_{-}+i \hat{\alpha}(I)\left(B_{+}+B_{-}\right)=0, \\
& \left( \pm \frac{\partial}{\partial X}+\frac{\partial}{\partial \tau}\right) B_{ \pm}+\sigma B_{ \pm}+i \hat{\alpha}(I)\left(A_{+}+A_{-}\right)=0,
\end{aligned}
$$

where $\hat{\alpha}(I)$ is some function of field intensity $I=|\vec{E}|^{2}$. It is easy to see that in spite of the fact that the set of Eqs. (29) is slightly different from that obtained in Ref. 26 it is clear that Eqs. (29) still describe $M$ solitons, which are significantly different from "conventional" Bragg solitons. Further study of the properties of the solitary waves supported by the 2D SPBG structures and described by Eqs. (29) is a subject for future research.

The 2D structures with "chessboard" corrugation pattern were studied and it was shown theoretically and experimentally that the corrugation with the "chessboard" pattern approximated well to the "ideal" sinusoidal corrugation. It was demonstrated that in accordance with theory the reflection coefficient at the exact Bragg frequency does not depend on the value of the structure length parameter $\alpha l_{z}$ and the minimum values of the transmission coefficients are the same for the zone associated with TEM $\leftrightarrow \mathrm{TEM}$ wave scattering. The experimental study of the 1D Bragg structure was also conducted. The measurement of the transverse rf field fluxes in the 2D SPBG structure with the "chessboard" pattern was also conducted. The results obtained from these experimental studies have been compared with the theoretical predictions and good agreement between the theoretical data and the experimental results has been demonstrated.

\section{ACKNOWLEDGMENTS}

The authors would like to thank Professor N. S. Ginzburg, Dr. N. Yu. Peskov, and Dr. A. S. Sergeev for useful discussions. The authors would also like to thank Professor G.G. Denisov for providing a wave transformer for the experiments. The authors would also like to thank EPSRC and the UK QINETIQ for partial support of this work.

\section{APPENDIX}

The perturbations induced by a shallow corrugation can be taken into account by considering the surface magnetic current $j^{m}$ on the unperturbed surface of the waveguide ${ }^{1}$

$$
\vec{j}^{m}=\frac{c}{4 \pi}\left[\vec{n} ; \nabla(r \vec{E} \vec{n})+i \frac{\omega}{c} r[\vec{n} \vec{H}]\right],
$$

where $\vec{n}$ is the normal unit vector directed towards the metal walls (outside of the unperturbed waveguide), $r$ is the function describing the periodic perturbation (1), $\vec{E}$ and $\vec{H}$ are the vectors of the electric and magnetic rf fields, $\omega$ is the wave frequency, and $\nabla$ is the gradient operator. Taking into account Eq. (A1), the waveguide excitation can be investigated for the approximation where the unperturbed waveguide eigenmode spatial structure is conserved along the radial coordinate. The rf electric and magnetic fields inside a $2 \mathrm{D}$ SPBG structure can be presented as ${ }^{30}$

$$
\vec{E}=e^{i \omega t} \sum_{l=1}^{4} C_{l} \vec{E}_{l}, \vec{H}=e^{i \omega t} \sum_{l=1}^{4} C_{l} \vec{H}_{l}
$$

where $C_{l}=C_{l}(z, x)$ is the slow function of the $z$ - and $x$-coordinate and

$$
\begin{aligned}
& \vec{E}_{1,2}=\vec{E}_{1,2}^{0}(r) e^{\mp i k_{z} z}, \vec{H}_{1,2}=\vec{H}_{1,2}^{0}(r) e^{\mp i k_{z} z}, \\
& \vec{E}_{3,4}=\vec{E}_{3,4}^{0}(r) e^{\mp i k_{x} x}, \vec{H}_{3,4}=\vec{H}_{3,4}^{0}(r) e^{\mp i k_{x} x} .
\end{aligned}
$$

Here, $\vec{E}_{l}^{0}, \vec{H}_{l}^{0}$ are the functions describing the wave spatial profile in a coaxial waveguide. The indices $l=1,2$ correspond to the waves propagating in the $\pm z$ directions, while indices $l=3,4$ correspond to the waves propagating in the $\pm x$ directions. To describe the wave scattering on the $2 \mathrm{D}$ Bragg corrugation let us use the new set of coordinates $(\xi, \eta, r)$ which can be obtained by rotating the original system coordinates $(z, x, r)$ such that

$$
x=\frac{1}{2 \overline{k_{x}}}(\xi-\eta), z=\frac{1}{2 \overline{k_{z}}}(\xi+\eta),
$$

and substituting the new variables in Eq. (1) one obtains

$$
r \cong r_{\text {in }}+a_{1}(\cos \xi+\cos \eta) \text {. }
$$


The two-dimensional periodic perturbation can be considered as a superposition of two one-dimensional corrugations (A5). As a result, the wave scattering can also be considered as waveguide mode excitation by two sets of surface magnetic currents $j_{k}^{m}$. Obviously, the first set of currents $j_{1}^{m}$ does not depend on the coordinate $\xi$ (due to the absence of perturbations along this coordinate) while the second set of the currents $j_{2}^{m}$ does not depend on the coordinate $\eta$. The coefficients $C_{l}$ satisfy equations: ${ }^{30}$

$$
\mathcal{L}_{l} C_{l}=-\frac{1}{N_{l, \xi}} \oint \vec{j}_{l}^{m} \vec{H}_{-l} d \sigma_{\xi}-\frac{1}{N_{l, \eta}} \oint \vec{j}_{2}^{m} \vec{H}_{-l} d \sigma_{\eta}
$$

where $N_{l, \xi}$ and $N_{l, \eta}$ are the norm of the interacting waves, $\mathcal{L}_{1,2}= \pm 2 \overline{k_{z}}(d / d \xi+d / d \eta), \mathcal{L}_{3,4}= \pm 2 \overline{k_{x}}(d / d \xi-d / d \eta)$. The integrals in Eq. (A6) are taken along closed contours $\sigma_{\xi, \eta}$ which are the crossings of the unperturbed surface of the waveguide conductors and the planes $\xi, \eta=$ const. Therefore the coefficients $C_{l}$ can be written as

$$
C_{l}(x, z)=C_{l}(\xi, \eta)=C_{l}^{\prime}(\xi)+C_{l}^{\prime}(\eta)
$$

Note, that Eqs. (A6) and (A7) indicate that both corrugations contribute independently and equally to the scattering of the partial waves. The expressions under the integrals (A6) can be brought to the following form:

$$
\vec{j}_{k}^{m} \vec{H}_{-l}=\frac{c}{4 \pi}\left[-\frac{i \omega}{c} r(\xi, \eta)\left(E_{n} E_{-l, n}+H_{\tau} H_{-l, \tau}\right)\right],
$$

where $r(\xi, \eta)$ is the function presented by Eq. (A5), indexes $n$ and $\tau$ indicate the normal and tangential components of the fields, respectively, $E_{n}, H_{\tau}$ are the normal electric and tangential magnetic fields of the wave incident on the corrugation, $k=1$, 2. Substituting the new coordinates into expressions (A1) one obtains

$$
\begin{aligned}
& \vec{E}_{1,2}=\vec{E}_{1,2}^{0}(r) e^{\mp i k_{z} / 2 \bar{k}_{z}(\xi+\eta)}, \\
& \vec{H}_{1,2}=\vec{H}_{1,2}^{0}(r) e^{\mp i k_{z} / 2 k_{z}(\xi+\eta)}, \\
& \vec{E}_{3,4}=\vec{E}_{3,4}^{0}(r) e^{\mp i k_{x} / 2 \bar{k}_{x}(\xi-\eta)}, \\
& \vec{H}_{3,4}=\vec{H}_{3,4}^{0}(r) e^{\mp i k_{x} / 2 k_{x}(\xi-\eta)},
\end{aligned}
$$

and substituting Eqs. (A8) and (A9) into Eq. (A6) one can obtain the following expression:

$$
\mathcal{L}_{l} C_{l}=-\frac{i \omega a_{1}}{8 \pi N_{l}}\left(\oint F(\xi, \eta) d \sigma_{\xi}+\oint F(\xi, \eta) d \sigma_{\eta}\right),
$$

where $N_{l}=c / 2 \pi \int_{a}^{a+a_{0}}\left[\vec{E}_{l}^{0} \vec{H}_{-l}^{0}\right] \vec{s}^{0} d r$ is the wave norm, $\vec{s}^{0}$ is the unit vector whose direction coincides with the direction of propagation of the rf power, and

$$
\begin{aligned}
F(\xi, \eta)= & \left(e^{i \xi}+e^{-i \xi}+e^{i \eta}+e^{-i \eta}\right)\left(\sum_{m=1}^{4}\left(C_{m}^{\prime}+C_{m}^{\prime \prime}\right) \vec{E}_{m, n}^{0}(r)\right. \\
& \times \vec{E}_{-l, n}^{0}(r) \times e^{i \varphi_{-l}+i \varphi_{m}}+\sum_{m=1}^{4}\left(C_{m}^{\prime}+C_{m}^{\prime \prime}\right) \\
& \left.\times \vec{H}_{m, \tau}^{0}(r) \vec{H}_{-l, \tau}^{0}(r) \times e^{i \varphi_{-l}+i \varphi_{m}}\right)_{r=a},
\end{aligned}
$$

where $\varphi_{j}$ refer to the partial waves' fast phase introduced in Eq. (A9), $\varphi_{j}=-\varphi_{-j}$. If the wave numbers $k_{z, x}$ are real the evaluation of the integrals (A10) along the closed contours will give nonzero results only if the function $F(\xi, \eta)$ can be represented as

$$
F(\xi, \eta)=G_{1}(\xi)+G_{2}(\eta),
$$

which can only be fulfilled if

$$
\frac{k_{x}}{2 \overline{k_{x}}}+\frac{k_{z}}{2 \overline{k_{z}}}-1=0 \quad \text { and } \frac{k_{x}}{\overline{\overline{k_{x}}}}=\frac{k_{z}}{\overline{k_{z}}} .
$$

Thus, considering Eqs. (A13) and (7), the Bragg resonance condition which is required to obtain $2 \mathrm{D}$ Bragg scattering can be written as

$$
\frac{k_{z}}{\overline{k_{z}}}=\frac{k_{x}}{\overline{k_{x}}} .
$$

Taking into account the expressions for $\vec{E}_{l}$ and $\vec{H}_{l}$ and Eq. (A12), Eq. (A10) can be rewritten in the following form:

$$
\mathcal{L}_{l} C_{l}=-i \overline{\alpha_{l}}\left(\left.G_{2}(\eta)\right|_{r=a}+\left.G_{1}(\xi)\right|_{r=a}\right),
$$

where $\overline{\alpha_{l}}=\omega a_{1} / 8 \pi N_{l}$ are the coupling coefficients and $N_{l}$ is the wave norm.

Taking into account the Bragg resonance conditions and the averaging over the fast period of the oscillations one can return to the original coordinate frame $(z, x, y)$ and obtain the following set of the coupled wave equations:

$$
\begin{aligned}
& e^{-i \Delta_{z} z} \frac{d C_{1}}{d z}=i\left(C_{3} \alpha_{1} e^{-i \Delta_{x} x}+C_{4} \alpha_{-1} e^{i \Delta_{x} x}\right), \\
& e^{i \Delta_{z} z} \frac{d C_{2}}{d z}=-i\left(C_{3} \alpha_{2} e^{-i \Delta_{x} x}+C_{4} \alpha_{-2} e^{i \Delta_{x} x}\right), \\
& e^{-i \Delta_{x} x} \frac{d C_{3}}{d x}=i\left(\alpha_{3} C_{1} e^{-i \Delta_{z} z}+\alpha_{-3} C_{2} e^{i \Delta_{z} z}\right), \\
& e^{i \Delta_{x} x} \frac{d C_{4}}{d x}=-i\left(\alpha_{4} C_{1} e^{-i \Delta_{z} z}+\alpha_{-4} C_{2} e^{i \Delta_{z} z}\right),
\end{aligned}
$$

where $\Delta_{x, z}=k_{x, z}-\bar{k}_{x, z}$ are the detuning differences from the Bragg resonance and $\alpha_{l}$ are the coupling coefficients. It is important to note, that if the waveguide walls are lined with a medium with a $2 \mathrm{D}$ biperiodic refractive index, the coupled wave equations similar to Eq. (A16) can be found by evaluating the Maxwell equations, under the geometric-optical approximation, when averaging over the fast period of the oscillations has been carried out. The rf field in this case should also be considered as a sum of four coupled waves. 
Let us assume that the grating has axial symmetry, i.e., the angles between the corrugations and the $z$ axis are the same. This assumption results in $\alpha_{l}=\alpha_{-l}$. Due to the homogeneity of the system, i.e., the "+" and " - " directions cannot be distinguished $\alpha_{1}=\alpha_{2}=\alpha_{z}$ and $\alpha_{3}=\alpha_{4}=\alpha_{x}$. Substituting $\mathcal{A}_{ \pm}$and $\mathcal{B}_{ \pm}$instead of $C_{l}$ one can obtain the set of equations describing the rf field scattering on the $2 \mathrm{D}$ Bragg corrugation

$$
\begin{aligned}
& e^{\mp i \Delta_{z} z} \frac{d \mathcal{A}_{ \pm}}{d z}= \pm i \alpha_{z}\left(\mathcal{B}_{+} e^{-i \Delta_{x} x}+\mathcal{B}_{-} e^{i \Delta_{x} x}\right), \\
& e^{\mp i \Delta_{x} x} \frac{d \mathcal{B}_{ \pm}}{d x}= \pm i \alpha_{x}\left(\mathcal{A}_{+} e^{-i \Delta_{z} z}+\mathcal{A}_{-} e^{i \Delta_{z} z}\right)
\end{aligned}
$$

where $\alpha_{z, x}$ are the coupling coefficients. Introducing a dimensionless detuning from the Bragg frequency $\hat{\delta}=\omega$ $-\omega_{0} / \omega_{0}, \Delta_{x, z}$ can be written as $\Delta_{z, x}=\nu_{z, x} \hat{\delta}$ where $\nu_{z}$ $=\omega_{0}\left(k_{z}-\overline{k_{z}} / \omega-\omega_{0}\right), \nu_{x}=\omega_{0}\left(k_{x}-\overline{k_{x}} / \omega-\omega_{0}\right)$, and considering ohmic losses $\sigma$ the coupled wave Eqs. (A17) can be presented for dimensionless parameters and variables in the following form:

$$
\begin{aligned}
& \frac{\partial A_{ \pm}}{\partial Z} \pm i \overline{\delta A_{ \pm}} \pm \overline{\sigma A_{ \pm}} \pm i \bar{\alpha}\left(B_{+}+B_{-}\right)=0, \\
& \frac{\partial B_{ \pm}}{\partial X} \mp i \bar{\delta} B_{ \pm} \pm \bar{\sigma} B_{ \pm} \pm i \bar{\alpha}\left(A_{+}+A_{-}\right)=0
\end{aligned}
$$

where $\quad X=x \omega_{0} / c \sqrt{\nu_{x} / \nu_{z}}, \quad Z=z \omega_{0} / c \sqrt{\nu_{z} / \nu_{x}}, \quad \bar{\delta}$ $=\hat{\delta} c / \omega_{0} \sqrt{\nu_{z} \nu_{x}}, \bar{\sigma}=\sigma c / \omega_{0} \sqrt{\nu_{z} \nu_{x}}$, and $\bar{\alpha}$ is the normalized coupling coefficient (see Ref. 17). It is important to note that if $\hat{\delta} \ll 1$, the expressions for $\nu_{z}$ and $\nu_{x}$ can be simplified to $\nu_{z}=\omega_{0}^{2} / c^{2} \overline{k_{z}}$ and $\nu_{x}=\omega_{0}^{2} / c^{2} \bar{k}_{x}$. Let us also note that the normalization coefficients of the partial waves' amplitudes ${ }^{17}$ are different and the coefficients can only be equal to each other if the structures of the partial waves are the same and $\nu_{x}$ $=\nu_{z}$, i.e., $\varphi=\pi / 4[$ Fig. $1(\mathrm{~b})]$.

${ }^{1}$ N. F. Kovalev, I. M. Orlova, and M. I. Petelin, Izv. Vyssh. Uchebn. Zaved., Radiofiz. 11, 783 (1968). (in Russian).

${ }^{2}$ A. Yariv and M. Nakamura, IEEE J. Quantum Electron. 13, 233 (1977).

${ }^{3}$ V. L. Bratman, G. G. Denisov, N. S. Ginzburg, and M. I. Petelin, IEEE J. Quantum Electron. 19, 282 (1983).

${ }^{4}$ E. Yablonovitch, Phys. Rev. Lett. 58, 2059 (1987).

${ }^{5}$ E. Yablonovitch and T. J. Gmitter, Phys. Rev. Lett. 63, 1950 (1989).

${ }^{6}$ T. S. Chu, F. V. Hartemann, B. G. Danly, and R. J. Temkin, Phys. Rev. Lett. 72, 2391 (1994).

${ }^{7}$ P. Zambon, W. J. Witteman, and P. J. M. Van der Slot, Nucl. Instrum. Methods Phys. Res. A 407, 413 (1998).
${ }^{8}$ A. W. Cross, N. S. Ginzburg, W. He, D. A. Jaroszynski, N. Yu. Peskov, A. D. R. Phelps, and C. G. Whyte, Nucl. Instrum. Methods Phys. Res. A 407, 181 (1998).

${ }_{9}^{9}$ J. Mathew and J. A. Pasour, Phys. Rev. Lett. 56(17), 1805 (1986).

${ }^{10}$ S. J. Cooke, A. W. Cross, W. He, and A. D. R. Phelps, Phys. Rev. Lett. 77, 4836 (1996).

${ }^{11}$ N. S. Ginzburg, A. A. Kaminsky, A. K. Kaminsky, N. Yu. Peskov, S. N. Sedykh, A. P. Sergeev, and A. S. Sergeev, Phys. Rev. Lett. 84, 3574 (2000).

${ }^{12}$ D. Eisert, G. Bacher, M. Legge, A. Forchel, J. Numberger, K. Schull, W. Faschinger, and G. Landwehr, Appl. Phys. Lett. 71, 1026 (1997).

${ }^{13}$ J. M. Lupton, B. J. Materson, I. D. W. Samuel, M. J. Jory, and W. L. Barnes, Appl. Phys. Lett. 77, 3340 (2000).

${ }^{14}$ N. S. Ginzburg, A. S. Sergeev, N. Yu. Peskov, G. R. M. Robb, and A. D. R. Phelps, IEEE Trans. Plasma Sci. 24, 770 (1996).

${ }^{15}$ N. F. Kovalev, V. E. Nechaev, M. I. Petelin, N. I. Zaitsev, International Workshop on the High Power Microwave Generation and Pulse Shortening (Digest of Technical Papers, EICC, Edinburgh, Scotland, 1997), p. 345 .

${ }^{16}$ N. S. Ginzburg, N. Yu. Peskov, and A. S. Sergeev, Opt. Commun. 96, 254 (1993).

${ }^{17}$ N. S. Ginzburg, N. Yu. Peskov, A. S. Sergeev, A. D. R. Phelps, I. V. Konoplev, G. R. M. Robb, A. W. Cross, A. V. Arzhannikov, and S. L. Sinitsky, Phys. Rev. E 60, 935 (1999).

${ }^{18}$ N. S. Ginzburg, I. V. Konoplev, A. S. Sergeev, Tech. Phys. 66, 108 (1996); N. S. Ginzburg, N. Yu. Peskov, A. S. Sergeev, I. V. Konoplev, A. W. Cross, A. D. R. Phelps, G. R. M. Robb, K. Ronald, W. He, and C. G. Whyte, J. Appl. Phys. 92, 1619 (2002).

${ }^{19}$ N. S. Ginzburg, A. S. Sergeev, N. Yu. Peskov, I. V. Konoplev, G. R. M. Robb, and A. D. R. Phelps, Nucl. Instrum. Methods Phys. Res. A 375, 202 (1996).

${ }^{20}$ A. W. Cross, W. He, I. V. Konoplev, A. D. R. Phelps, K. Ronald, G. R. M. Robb, C. G. Whyte, N. S. Ginzburg, N. Yu. Peskov, and A. S. Sergeev, Nucl. Instrum. Methods Phys. Res. A 475, 164 (2001).

${ }^{21}$ N. V. Agarin, A. V. Arzhannikov, V. B. Bobylev, N. S. Ginzburg, V. G. Ivanenko, P. V. Kalinin, S. A. Kuznetsov, N. Yu. Peskov, A. S. Sergeev, S. L. Sinitsky, and V. D. Stepanov, Nucl. Instrum. Methods Phys. Res. A 445, 222 (2000).

${ }^{22}$ S. Fan, P. R. Villeneuve, J. D. Joannopoulos, and H. A. Haus, Phys. Rev. Lett. 80, 960 (1998).

${ }^{23}$ S.G. Johnson, S. Fan, P. R. Villeneuve, J. D. Joannopoulos, and L. A. Kolodziejski, Phys. Rev. B 60, 5751 (1999).

${ }^{24}$ M. Agio and L. C. Andreani, Phys. Rev. B 61(23), 15519 (2000).

${ }^{25}$ J. R. Sirigiri, K. E. Kreischer, J. Machuzak, I. Mastovsky, M. A. Shapiro, and R. J. Temkin, Phys. Rev. Lett. 86, 5628 (2001).

${ }^{26}$ Neşet Aközbek and Sajeev John, Phys. Rev. E 57, 2287 (1998).

${ }^{27}$ A. F. Harvey, Microwave Engineering (Academic, London, 1963).

${ }^{28}$ I. V. Konoplev, A. W. Cross, W. He, A. D. R. Phelps, K. Ronald, G. R. M Robb, C. G. Whyte, N. S. Ginzburg, N. Yu. Peskov, and A. S. Sergeev, Nucl. Instrum. Methods Phys. Res. A 445, 236 (2000).

${ }^{29}$ G. G. Denisov, M. G. Reznikov, Radiophys. Quantum Electron. (Izv. Vyssh. Uchebn. Zaved., Radiofiz.) 15, 562 (1982).

${ }^{30}$ D. V. Vinogradov, G. G. Denisov, Int. J. Infrared Millim. Waves 12, 131 (1991).

${ }^{31}$ L. A. Vainshtein, Electromagnetic Waves, 2nd ed. (Radio i Svyaz, Moscow, 1988) [in Russian]. 